\title{
IP routing by phase induced intensity noise suppression in Optical CDMA network
}

\author{
C. B. M. Rashidi ${ }^{1, ~ *}$, S. A. Aljunid ${ }^{1}$, M. S. Anuar ${ }^{1}$, Hassan Yousif Ahmed ${ }^{2}$ \\ ${ }^{1}$ Centre of Excellence Advanced Communication Engineering, School of Computer and Communication Engineering, UniMAP, Kangar, Perlis, \\ Malaysia \\ ${ }^{2}$ Salman bin Abdul Aziz University, Al Kharj, Saudi Arabia
}

Email address:

rashidibeson@unimap.edu.my (C. B. M. Rashidi)

\section{To cite this article:}

C. B. M. Rashidi, S. A. Aljunid, M. S. Anuar, Hassan Yousif Ahmed. IP Routing by Phase Induced Intensity Noise Suppression in Optical CDMA Network. American Journal of Networks and Communications. Special Issue: Recent Progresses in Optical Code-Division Multiple-Access (OCDMA) Technology. Vol. 4, No. 2-1, 2015, pp. 18-21. doi: 10.11648/j.ajnc.s.2015040201.13

\begin{abstract}
In this paper, we explore avenues to efficiently use spectral amplitude coding optical code-division multiple-access (SAC-OCDMA) technique as a solution to the Internet protocol (IP) routing bottleneck in the global internet access. This work reveals for the first time the authors proposed a novel class of codes called the flexible cross correlation (FCC) code to enhance the network capacity and achieve the highest performance possible through phase induced intensity noise (PIIN) evasion and eliminating multiple access interference (MAI). The results indicated good performance, where the FCC code can accommodate 150 simultaneous users and has an optimum receive power $P_{s r}=-25 \mathrm{dBm}$ at the receiving plant. It is truly proved that, from the theoretical results, the SAC-OCDMA technique can be an excellent candidate for the future IP backbone.
\end{abstract}

Keywords: PIIN, Flexible Cross Correlation Code, Internet Protocol, MAI, SAC-OCDMA, MPLS, FTTx

\section{Introduction}

Internet protocol (IP) has been the dominant protocol to deliver the recent multimedia-on-demand services and also predicted to be for the future backbones using a fiber-distributed data interface in next generation networks sub-scribers such as fiber-to-the-home/curb/node (FTTx) [1]. Furthermore, to support a huge number of simultaneous users on high bit-rate services, an expansion in network capacity leading to the higher data traffic is required. In addition, IP routing operates in the electrical domain in the network layer, thus, it cannot be processed at a speed matched the huge transmission speed offered by the fiber optic [2]. As a solution to this problem, multiple-protocol label switching (MPLS) has been proposed by [3], where each packet is assigned a label at the ingress edge router. At the intermediate nodes, a packet is forwarded only according to its label. Since network layer label analysis is avoided, significant processing time is saved at each hop. Also the end-to-end delay can be dramatically reduced because IP routing is only needed at the edge of the core network [4]. Nevertheless, MPLS partially relieves the burden of IP routing; the electrical routing scheme will still become a bot-tleneck as IP traffic increases. All-optical packet switching might be another solution to this problem, by the use of pure optical signal processing. However, there are still many difficulties in its implementation, such as time synchronization, contention resolution and optical random access memory (RAM) [5]. To mitigate the problem of traffic growth and need for enhanced multiple user access to the network resources, optical code-division multiple-access (OCDMA) has been introduced for more than a decade [1]. The great advantage of such a system lies in the fact that multiple users can access the network asynchronously, simultaneously and securely [6]. However, multiple access interference (MAI) is the main performance degradation especially when large numbers of users are involved in OCDMA systems. Since, among all encoding approaches were very difficult to sustain in optical fiber, thus spectral amplitude coding (SAC) with the incoherent signal of the same coherent version was proposed [7]. SAC has the advantages of eliminating the effect of MAI when code with flexibility cross-correlation property is utilized as address sequences and balance detection at the receiver (see Figure 1). In SAC-OCDMA system, phase induced intensity noise (PIIN) is deeply related to MAI due to overlapping spectra from different users. Inappropriate cross-correlation among the 
address sequences will cause PIIN between code sequences increased [8]. One of the effective solutions for suppression the effect of PIIN is, decreasing the number of interferences between the signals of different users with an effective designing a code with high peak auto-correlation as well as low side lobe cross-correlation in SAC-OCDMA system [9]. Most codes have been proposed for the SAC-OCDMA systems such Dynamic Cyclic Shift (DCS) [7], Modified Frequency Hopping (MFH) [8] and Modified Double Weight (MDW) codes, respectively [10]. However, these codes have several limitations such as the code construction is complicated (e.g. DCS code), the code is either too long (e.g. Hadamard) and an ideal cross-correlation for MFH code. In this paper, a new coding algorithm named FCC code is proposed to improve system capacity and achieve higher performance possible through suppressing PIIN and eliminating MAI. The proposed code also has an advantage of high cardinality and low received power with shorter code length.

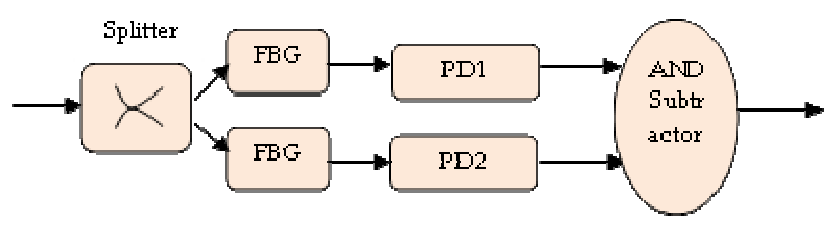

Figure 1. Structure of a MAI-alleviation decoder [7].

\section{Algorithm for FCC Code Development}

Optical codes are family of $K$ (for $K$ users) binary " 0 " and " 1 " sequences of length $N$, code weight $W$ (the number of " 1 " in each codeword) and the maximum cross-correlation, $\lambda_{\max }$. In OCDMA system, to allow receivers to distinguish each of the possible users, to reduce channel interference and to accommodate large number of users, optical codes should have large values of $W$ and the size $K$.

Step 1:

The set optical code consists of $\left(N, W, \lambda_{\text {max }}\right)$ FCC code for $K$ users. The $K \times N$ code matrix is here called the Tridiagonal Code Matrix. These sets of codes are then represented by;

$$
A_{K}^{W}=\left[\begin{array}{ccccccc}
a_{11} & a_{12} & a_{13} & 0 & 0 & \cdots & 0 \\
a_{21} & a_{22} & a_{23} & a_{24} & 0 & \cdots & \vdots \\
0 & a_{32} & a_{33} & a_{34} & a_{35} & 0 & \vdots \\
0 & 0 & a_{43} & a_{44} & a_{45} & a_{46} & \vdots \\
\vdots & \ddots & \ddots & \ddots & \ddots & \ddots & \vdots \\
0 & 0 & \cdots & \cdots & \cdots & \cdots & a_{K N}
\end{array}\right]=\left[\begin{array}{c}
A_{1} \\
A_{2} \\
A_{3} \\
\vdots \\
\vdots \\
A_{K}
\end{array}\right]
$$

$$
\text { where, } \begin{gathered}
A_{1}=a_{11}, a_{12}, a_{13} \ldots a_{1 N} \\
A_{2}=a_{21}, a_{22}, a_{23}, a_{24} \ldots a_{2 N} \\
A_{3}=a_{31}, a_{32}, a_{33}, a_{34} \ldots a_{3 N} \\
\vdots \\
A_{K}=a_{K 1}, a_{K 2}, a_{K 3} \ldots a_{K N}
\end{gathered}
$$

The rows of $\mathrm{A}_{1}, \mathrm{~A}_{2}$ and $\mathrm{A}_{k}$ represent the $K$ codeword and it is assumed that, the code weight of each of the $K$ codeword is to be $W$.

Step 2:
After the $K$ codes represented by the $K$ rows of the $K \times N$ code matrix in Equation (1), are to represent a valid set of $K$ codeword with in phase cross-correlations $\lambda_{\max }$ and code weight $W$; it must satisfy the following conditions:

1 . The elements $\left\{\mathrm{a}_{\mathrm{ij}}\right\}$ of must have values " 0 " or " 1 "

$$
\mathrm{a}_{\mathrm{ij}}=\text { " } 0 \text { " or " } 1 \text { " for } \mathrm{i}=1,2, . . K, \mathrm{j}=12, . . N
$$

2 . The in phase cross-correlation $\lambda_{\max }$, between any of the $K$ code words ( $K$ rows of the matrix ) should not exceed code weight $W$. That is,

$$
X_{i} X_{j}^{T}= \begin{cases}\leq \lambda_{\max } & \text { for } i \neq j \\ =W & \text { for } i=j\end{cases}
$$

3. The code weight of each codeword should be equal to $W$ where,

$$
\sum_{j=1}^{N} a_{i j}=W, \quad i=1,2 \ldots K
$$

4. From equation (3), it is seen that the $W=X_{\mathrm{i}} X_{\mathrm{i}}^{\mathrm{T}}$ is the in phase auto-correlation function of codes. $X_{\mathrm{i}} Y_{\mathrm{j}}^{\mathrm{T}}$ is the out of phase cross-correlation between the $i^{\text {th }}$ and the $j^{\text {th }}$ codes. It follows that $X_{\mathrm{i}} X_{\mathrm{i}}^{\mathrm{T}}$ should be greater than $X_{\mathrm{i}} Y_{\mathrm{j}}^{\mathrm{T}}$. In other words, $W>\lambda_{\max }$

5. All $K$ rows of should be linearly independent because each codeword must be uniquely different from other codewords. That is to say the rank of the $K \times N$ matrix should be $K$. Moreover, for to have $\operatorname{rank} K$, thus codes $N \geq K$.

Step 3:

From the five conditions above in Step 2, one of the matrices binary sequences as shown in equation (1) in Step 1, whose the first $i^{\text {th }}$ row for the first $K$ user is given by;

$$
A_{i}=\overbrace{0 \ldots 0}^{r(i-1)} \overbrace{11 \ldots 1}^{W} \quad \overbrace{0 \ldots 0}^{r(K-i)}
$$

The length $N$ of the codes which is the length of the rows of the $K \times N$ code matrix is given by;

$$
N=W K-\lambda_{\max }(K-1)
$$

It can be seen that the length $N$ is minimum under the assumed conditions.

\section{Performance Analysis}

In our analyses, we only considered shot noise $\left\langle i_{\text {shot }}>\right.$, incoherent intensity noise $\left\langle i_{\text {PIIN }}>\right.$ and thermal noise $\left\langle i_{\text {thermal }}>\right.$ to evaluate the system performance. The SNR is defined as the average of the signal-to-noise ratio, $\mathrm{SNR}=\left[\mathrm{I}^{2} / \sigma^{2}\right]$ where $\sigma^{2}$ is the mean power of noise which is given by;

$$
\sigma^{2}=2 e B I+I^{2} B \tau_{C}+\frac{4 K_{b} T_{n} B}{R_{L}}
$$

where, $e$ is the electron's charge, $I$ is the average photocurrent, $I^{2}$ is the power spectral density for $I, B$ is the noise equivalent 
ofelectrical bandwidth, $K_{b}$ is the Boltzmann constant, $T_{n}$ is theabsolute receiver noise temperature, $R_{L}$ is the receiver load resistor and $\tau_{\mathrm{c}}$ is the coherence source time. Only one power spectral density (PSD) spectrum will be calculated and the photodiode current $I$ can be written as follows;

$$
I=\Re \int_{0}^{\infty} G(v) d v
$$

represents as the responsivity of the photo-detectors. Consequently, the photo current $I$ can be expressed as;

$$
I=\mathfrak{R}\left[\frac{P_{s r} W}{N}\right]
$$

The mean power of shot noise can be written as;

$$
I_{\text {shot }}=2 e B \Re\left[\frac{P_{s r}}{N}\right][W+3]
$$

We assume that, the intensity noise will dominate the broadband sources. Hence, with power spectral density from each user is the same; therefore, we calculate the receiver intensity noise directly from the total power spectral density of each photodiode and the summation of

$$
\sum_{m=1}^{K} d_{m}(i) c_{m}(i) \approx \frac{K W}{N}
$$

the variance of the receiver photocurrent can be expressed as;

$$
I_{P I I N}^{2}=\frac{B \Re^{2} P_{s r}^{2} K W}{N^{2} \Delta v}[W+3]
$$

Thermal noise is given as [11];

$$
I_{T N}=\frac{4 K_{b} T_{n} B}{R_{L}}
$$

From equations (9), (10), (11) and (12) the SNR for the proposed FCC code SAC-OCDMA coding systems is defined by the mathematical expression as follows;

$$
S N R=\frac{\left[\frac{\Re P_{s r} W}{N}\right]^{2}}{\left[\frac{2 e B \Re P_{s r}}{N}\right][W+3]+B \Re^{2}\left[\frac{P_{s r}{ }^{2} K W}{N^{2} \Delta V}\right][W+3]+\frac{4 K_{b} T_{n} B}{R_{L}}} .
$$

Since, there is no pulses are sent for the data spacing assuming that the noise distribution is Gaussian thus, the corresponding bit-error rate (BER) can be obtained as follows [7];

$$
P_{e}=\frac{1}{2} \operatorname{erfc}\left(\sqrt{\frac{S N R}{8}}\right)
$$

Finally, the equations (13) and (14) will be used for the numerical calculation for an evaluation of the proposed coding system using FCC code.

\section{Results and Discussions}

We analyze the relationship between SNR and BER based on equations (13) and (14), respectively. The critical factor in evaluating a code sequence is to achieve an optimum (maximum) cardinality, effective receive power, Psr and bit rate. Due to that, we evaluate our proposed code according to the numerical parameters given in Table 1 to obtain optimum (maximum) cardinality, effective power receive and bit rate by satisfying the design constraints.

Table 1. Parameters used for theoretical calculations.

\begin{tabular}{ll}
\hline Parameters & Values \\
\hline PD quantum efficiency & $\eta=0.75$ \\
Spectral width of broadband light & $\Delta \lambda=3.75 \mathrm{THz}$ \\
Operating wavelength & $\lambda_{0}=1550 \mathrm{~nm}$ \\
Electrical bandwidth & $\mathrm{B}=80 \mathrm{MHz}, 311 \mathrm{MHz}$ \\
Data transmission rate & $\mathrm{R}_{\mathrm{b}}=155 \mathrm{Mbps}, 622 \mathrm{Mbps}$ \\
Rx Noise temperature & $\mathrm{T}_{\mathrm{n}}=300 \mathrm{~K}$ \\
Receiver load resistor & $\mathrm{R}_{\mathrm{L}}=1030 \Omega$ \\
Boltzmann's constant & $K_{\mathrm{b}}=1.38 \times 10^{-23} \mathrm{~W} / \mathrm{K} / \mathrm{Hz}$ \\
Electron charge & $e=1.60217646 \times 10^{-19}$ coulombs \\
Light velocity & $C=3 \times 10^{8} \mathrm{~m} / \mathrm{s}$ \\
\hline
\end{tabular}

We observe from Figure 2 the variation plots of the number of simultaneous users versus system performance BER for FCC code $(W=4)$ with existing SAC-OCDMA codes such as $\operatorname{DCS}(W=4), \operatorname{MDW}(W=4), \operatorname{MFH}(W=4)$ and Hadamard $(\mathrm{W}=8)$ codes, respectively at $155 \mathrm{Mbps}$ bit rate. It can be seen that, the system performance BER degrade as the number of simultaneous users increased. At performance analysis BER $=$ $10^{-9}$ the system with FCC code $(W=4)$ can accommodate 150 numbers of simultaneous users, which is the highest cardinality as compare to DCS ( $W=4, K=90)$, MDW ( $W=4$, $K=55), \operatorname{MFH}(W=4, K=20)$ and Hadamard $(\mathrm{W}=8, \mathrm{~K}=15)$ codes. The percentage number of simultaneous users (cardinality) improvements are $66 \%, 172 \%, 650 \%$ and $900 \%$ as a contrast to $\operatorname{DCS}(W=4, K=90)$, MDW ( $W=4, K=55)$, MFH ( $W=4, K=20)$ and Hadamard $(W=8, K=15)$ codes, respec-tively. We can ascertain from this fact, that, the FCC code had indicated good performance due to arrangement of code algorithm and flexibility in phase cross-correlation.

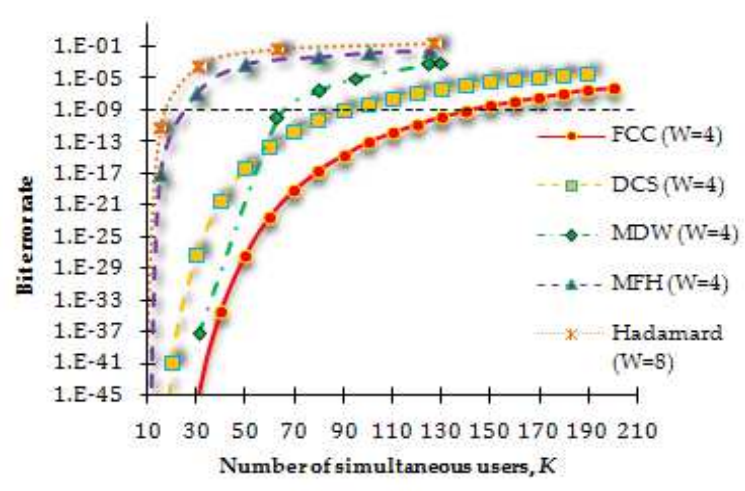

Figure 2. Number of simultaneous users versus system performance BER for various $S A C-O C D M A$ codes. 


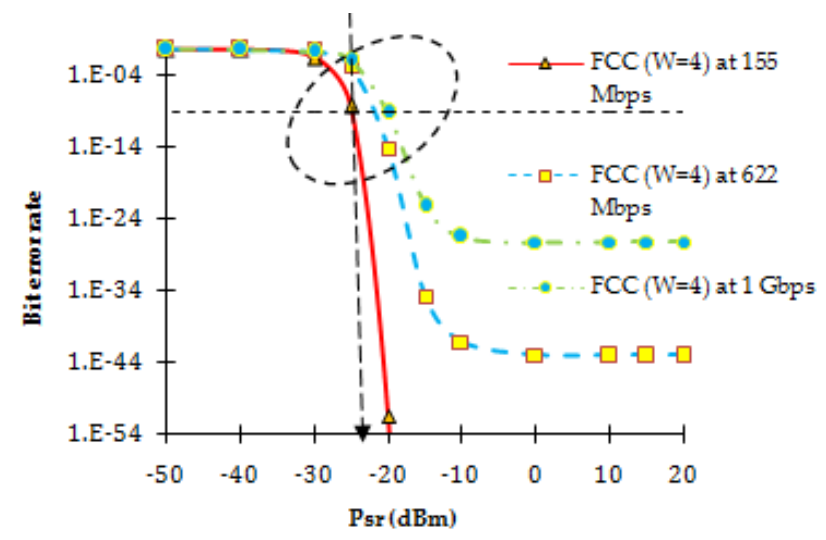

Figure 3. Effect of $P_{s r}$ on system performance for different $155 \mathrm{Mbps}, 622$ Mbps and 1 Gbps bit rate.

We observe Figure 3 the plots of receive power $P_{s r}$ versus system performance BER for FCC $(W=4)$ code utilizing MAI-alleviation decoder as shown in Figure 1 in our system. This evaluation is to see the effect on receive power Psr of the FCC $(W=4)$ code at different bit rates which are $155 \mathrm{Mbps}$, $622 \mathrm{Mbps}$ and $1 \mathrm{Gbps}$. From this figure, at $155 \mathrm{Mbps}$ utilizing MAI-alleviation decoder, FCC $(W=4)$ code can have an optimum value of a $P_{s r}=-25 \mathrm{dBm}$ without requiring any amplification. At system performance BER $10^{-9}, \mathrm{FCC}(W=4)$ code at $622 \mathrm{Mbps}$ and $1 \mathrm{Gbps}$ only indicate minimum receive power are $P_{s r}=-19 \mathrm{dBm}$ and $-18 \mathrm{dBm}$, respectively. There are about $-32 \mathrm{~dB}$ and $-33 \mathrm{~dB}$ effective receive power margins between them.

\section{Conclusions}

The FCC code properties and construction has been derived and presented for the IP solution backbone. The results of the BER performance of a SAC-OCDMA system utilizing FCC code also presented. The theoretical results indicated that the proposed FCC code achieves a highest number of simultaneous users and the FCC SAC-OCDMA may be one of the solutions for the IP backbone in the future of fast internet access.

\section{Acknowledgement}

This project was supported by the Deanship of Scientific Research at Salman bin Abdulaziz University under the research project\# 2014/1/877.

\section{References}

[1] M. Massoud Karbassian and H. Ghafouri-Shiraz, "IP Routing and Transmission Analysis in Optical CDMA Networks: Coherent Modulation With Incoherent Demodulation," Journal Of Lightwave Technology, vol. 27, no. 17, pp. 3845-3852, Sept. 2009.

[2] M. Massoud Karbassian and H. Ghafouri-Shiraz, "IP Routing and Traffic Analysis in Coherent Optical CDMA Networks," Journal Of Lightwave Technology, vol. 27, no. 10, pp. 1262-1268, May 2009.

[3] Mohammad Mehdi Shahsavari, "MPLS Performance Modeling Using Traffic Engineering to Improve QoS Routing on IP Networks," Proc. IEEE SoutheastCon, pp. 152-157, Apr. 2002.

[4] Zou Wei and H. Ghafouri-Shiraz, "IP routing by an optical spectral-amplitude-coding CDMA network," IET Journals, vol. 149, no. 56, pp. 265-269, Oct. 2002.

[5] Shun Yao, Yoo, S. J. B., Mukherjee, B., and Dixit, S., "All-optical packet switching for metropolitan area networks: opportunities and challenges," IEEE Journals \& Magazines, vol. 39 , no. 3, pp. 142-148, Aug. 2001.

[6] Salehi, J.A., "Code division multiple access techniques in optical fiber network Part 1: Fundamenial principles," IEEE Trans. Communications, vol. 37, no. 8, pp. 824-834, Aug. 1989.

[7] T. H. Abd, S. A. Aljunid, H. A. Fadhil, R. B. Ahmad, and M. A. Rashid, "New Approach for Evaluation of The Performance of Spectral Amplitude Coding-Optical Code Division Multiple Access System On High-Speed Data Rate," IET Communications, vol. 6, no. 12, pp. 1742-1749, Aug. 2012.

[8] Zou Wei and H. Ghafouri-Shiraz, "Unipolar Codes With Ideal In-Phase Cross-Correlation for Spectral Amplitude-Coding Optical CDMA Systems," IEEE Transaction on Communications, vol. 50, No. 8, pp. 1209-1212, Aug. 2002.

[9] Hamza M.R. Al-Khafaji, Razali Ngah, S.A. Aljunid, and T.A. Rahman, "A new two-code keying scheme for SAC-OCDMA systems enabling bipolar encoding," Journal of Modern Optics, 2014.

[10] S. A. Aljunid, M. D. A. Samad, M. Othman, M. H. Hisham, A. H. Kasiman, and M. K. Abdullah, "Development of modified double-weight code and its implementation in multi-rate transmissions," Proc. IEEE International Conference on Networks, Communication, 1, 288-292, Nov. 2005.

[11] Bartolo, R. E., Tveten, A. B., and Dandridge, A., "Thermal Phase Noise Measurements in Optical Fiber Interferometers," IEEE Journal of Quantum Electronics, vol. 48, no. 5, 720 727, March 2012. 